\title{
A Dynamic Model of Quadriceps and Hamstrings Function
} Carlo A. Frigo 1, Esteban E. Pavan 1, Reinald Brunner 2

1 Movement Biomechanics and Motor Control Laboratory

Department of Bioengineering, Politecnico di Milano, Milano, Italy

2 Neuro-Orthopaedic Department and the cLaboratory for Movement Analysis, University Children's Hospital, Basel, Switzerland

http://www.sciencedirect.com/science/article/pii/S0966636209006195 http://dx.doi.org/10.1016/j.gaitpost.2009.09.006

Correspondence to:

Prof. Eng. Carlo A. Frigo

Department of Electronic, Information and Bioengineering

Politecnico di Milano

Via Golgi 39

20133 Milano, Italy

Tel.: +39-02.2399.3346/9009

E-mail: carlo.frigo@polimi.it 


\title{
A Dynamic Model of Quadriceps and Hamstrings Function
}

\author{
Carlo A. Frigo, Esteban E. Pavan, Reinald Brunner
}

\section{Introduction}

The function of quadriceps and hamstrings during gait is complex. When the lower limb is weight bearing the hamstrings act more as hip extensors by controlling the position of the pelvis in space rather than as knee flexors [1, 2]. When forward lean of the trunk is used to obtain an external knee extension moment, the hamstrings, which contribute by stabilising the pelvis, in that sense can function as knee extensors as well. Co-contraction of quadriceps and hamstrings can be observed in normal situations, especially in stance phase, and is also seen in pathological gait. In particular in cerebral palsy (CP) co-contraction results in a increased knee stiffness that can occur at any time during gait and is usually attributed to inadequate and spastic muscle control. However, co-contraction of the quadriceps and hamstrings is also found in neurologically normal patients, especially those with reduced muscle strength [3]. Thus, other mechanisms than pathological muscle control may explain these observations. For example it is likely that knee extensors activity enhances the biarticular effect of the hamstrings as hip extensors. But rectus femoris, a component of quadriceps, acts as hip flexor as well so that the effect of quadriceps and hamstrings co-contraction on knee and hip kinematics will depend on the respective lever arms, instantaneous configuration of the joints, external loads and muscle recruitment.

In view of the foregoing a whole-body biomechanical model was developed to investigate the effects of simulated contraction of quadriceps and hamstrings on movement at the knee and hip joints. 


\section{Method}

\section{The model implementation}

The model adopted was derived from one described previously [4] and consisted of 13 rigid body segments connected each other by constraints. The rigid body segments represent head, trunk, pelvis, forearms and arms, thighs, shanks and feet. The total number of degrees of freedom (dof) was 32 . With reference to the only parts of interest for the present application, trunk and pelvis were connected by a spherical joint on slot, which allowed 3 relative rotations and sliding along an almost vertical line. This movement was controlled by four visco-elastic springs connecting trunk and pelvis. The hip was represented by a spherical joint (3 dof), while the ankle was defined as a revolute joint (1 dof). The knee was modelled as a multiple axes mechanism aiming at reproducing the cruciate ligament constraint. Two rigid bars were hinged to distal femur and proximal tibia and had the same length and orientation of the two cruciate ligaments $[5,6,7]$. The resulting relative movement was a rotation around a movable axis (1 dof) defined by the intersection of the two cruciate ligaments. The crossing bars mechanism was checked against a fixed axis flexion/extension mechanism and it was found that at $90^{\circ}$ of knee flexion the proximal tibia was shifted forward in relation to the position obtained by a single axis constraint by approximately $3 \mathrm{~cm}$, which is consistent with the well known gliding movement associated to tibio-femoral rotation. Geometric parameters were derived from MRI images from a healthy adult volunteer [8]. The volume of interest, from the lumbar region to tip toes, was scanned with a resolution of $1 \mathrm{~mm}$. All slices were processed 
using an image processing software (Amira-Mercury Computer Systems) to obtain a $3 \mathrm{D}$ reconstruction of the pelvis, femur, patella, tibia, and fibula. Finally, these virtual bones were included in a dynamic model, which was implemented in Working Model/VisualNastran (MSC-Software) (Figure 1).

\section{<<Insert Figure 1 here >>}

The following muscles were modelled: iliacus, psoas, rectus femoris, vasti, gluteus maximus and medius, semimembranosus, semitendinosus, biceps femoris (long and short heads), adductor magnus, gastrocnemius, soleus and tibialis anterior. Normally most of these muscles wrap over bone surfaces. In the model their tendons were modelled as a series of longitudinally oriented cylinders that glided over cylindrical surfaces. The contractile part of the muscles was modelled as a spring-damper element.

The patella, represented as a parallelepiped, was connected to the tibial tuberosity by a non extendible rope and could glide over the femoral trochlea. Gravitational field was set to zero to prevent the model to collapse on the floor. Segment masses were obtained from Clauser et al. [9] and moments of inertia were computed by considering a homogeneous density of the body segments. A pre-tension $(0.1 \mathrm{~N})$ was applied to the springs to enable the muscles to contact their respective wrap surfaces. A damping factor $(0.5 \mathrm{Ns} / \mathrm{m})$ was attributed to all muscles to obtain a distributed damping effect and prevent oscillations of the system. The force retained in the spring-damper elements could be seen as a representation of the basal tone of the muscles, and did not produce any appreciable movement to the system. 


\section{The simulation}

Muscle activity was simulated by an abrupt change of the rest length of the springdamper element representing the muscle of interest. In order to allow comparing the effects of different muscle contractions, every muscle was set to contract with the same initial force $(F)$ and to produce the same amount of mechanical work (W). As a consequence the length variation $(\Delta \mathrm{L})$ should also be the same. Looking at the geometry of the system, we decided that a feasible range of muscle length for all the muscles could be $0.02 \mathrm{~m}$. The amount of mechanical work could be $0.25 \mathrm{~J}$. Taking into account that $\mathrm{W}=1 / 2 \mathrm{~K} \Delta \mathrm{L}^{2}$, the elasticity constant $\mathrm{K}$ was equal to $1250 \mathrm{~N} / \mathrm{m}$ and the initial force $\mathrm{F}=\mathrm{K} \Delta \mathrm{L}=25 \mathrm{~N}$. This value was feasible by all the muscles considered. In fact, for example, by hypothesizing a physiologic cross sectional area (PCSA) of $20 \mathrm{~cm}^{2}$ and a specific maximum force of $15 \mathrm{~N} / \mathrm{cm}^{2}[10,11]$ the force produced by that muscle would be $300 \mathrm{~N}$, that is 12 times higher than the force we attributed to our muscles. The configuration of the multiple-body system obtained when the muscle achieved its rest length was considered the result of the muscle contraction. In most simulations the time required was betwee 0.4 and $0.7 \mathrm{~s}$. In some circumstances, when antagonist muscles were contracted, muscle shortening was extremely slow. If the muscle length was changed by less than $0.005 \mathrm{~m}$ after one second of simulation the simulation was stopped.

As the objective of this study was to investigate the reasons for co-contraction of knee extensors and hamstrings in principle, from the complex arrangement of muscles at hip and knee we selected the main functional components only: a) the biarticular group of hamstrings connecting pelvis to shank posteriorly represented by the Semimembranosus (SM), b) the monoarticular component of quadriceps connecting femur to shank anteriorly represented by one single vastus (VA), and c) 
the biarticular component of quadriceps connecting pelvis to shank anteriorly represented by Rectus Femoris (RF).

Single contractions of each muscle, and different combinations of them were considered. The initial conditions were defined as follows:

- Standing upright (pelvis, thigh and shank aligned vertically), with three possible conditions: 1) all segments free to move (as permitted by the joint constraints), 2) the pelvis free to move while the foot was fixed on the ground (representing stance phase), and 3) the foot free to move while the pelvis was fixed in space (representing swing phase);

- Different degrees of hip and knee flexion while the pelvis was vertical and all the segments were free to move according to their respective dof. The set of joint angles for the hip was $90^{\circ}, 60^{\circ}, 30^{\circ}, 0^{\circ},-15^{\circ}$, while for each hip position the set of knee angles was $30^{\circ}, 60^{\circ}, 90^{\circ}, 120^{\circ}$.

\section{Results}

The results of our simulation, with reference to standing initial position, are reported in Figure 2.

$<<$ Insert Figure 2 here >>

Contraction of SM alone produced hip extension and knee flexion when all body segments were free to move, but the effect of SM contraction at the hip switched from extension to slight flexion when the pelvis was fixed in space $(\mathrm{SM}(\mathrm{pf}))$. In this condition knee flexion was considerably increased. When the foot was fixed on the 
ground (SM(ff)), hip extension increased and knee flexion decreased considerably. When VA was contracted simultaneously with SM the knee extended by only few degrees, because it was close to full extension, but the hip extension was considerably enhanced. This occurred in all the three conditions considered. When RF contraction was added (not reported in the figure) slight hip abduction and external rotation was observed after several seconds of simulation, but there was no flexion-extension movement. Hence the effect of this combination of muscle contractions was not further considered.

$<<$ Insert Figure 3 here $>$

Figure 3 shows an example of the effect of the combined contraction of SM, VA and RF with an initial position of $60^{\circ}$ of flexion at the hip and knee joints. It can be observed that whenever VA activity was superimposed to SM activity, both the hip and the knee extended considerably. The activity of RF, superimposed on SM activity did not produce any relevant movement because of co-contraction, so that we stopped the simulation after one second (see Figure 3).

$$
\text { <<Insert Figure } 4 \text { here>> }
$$

In Figure 4 the effects of muscular contractions at different initial positions are compared. The first set of graphs (left most column, hip above, knee below) refer to a hip joint flexed at $90^{\circ}$ and the knee flexed at $30^{\circ}, 60^{\circ}, 90^{\circ}$ and $120^{\circ}$ respectively. RF contraction produced hip extension and knee extension, SM contraction produced hip extension and knee flexion, and VA contraction produced hip extension and knee extension. The combination of SM and VA contraction produced a considerable increase in hip extension, and this effect was more noticeable as knee flexion 
increased. Furthermore the knee flexion obtained with single SM contraction dramatically switched to knee extension. A similar effect, although not so noticeable, occurred with the combination of SM, VA and RF contractions. Simultaneous contraction of SM and RF for the joint angle combination hip flexion $90^{\circ}$ and knee flexion $120^{\circ}$ produced a few degrees of hip extension but this movement was very slow and the simulation was stopped after one second.

The other columns in Figure 4 refer to a hip joint progressively less flexed $\left(60^{\circ}, 30^{\circ}\right)$, and neutral and slightly extended $\left(0^{\circ},-15^{\circ}\right)$. For each hip position the same set of knee joint angles was considered, except in the last two positions where the knee joint angle was $30^{\circ}$. The effects of the different combinations of muscle contractions were qualitatively similar in the three columns, although the magnitude progressively increased, particularly for RF and SM plus VA contractions. The combination of SM and RF contractions was erratic because, with the exception of the first data point of $30^{\circ}$ of knee flexion, the simulation had to be stopped at $1 \mathrm{~s}$ because of the sluggishness of the movement. At $0^{\circ}$ and $15^{\circ}$ of hip extension, shown in the last data column, the effects of muscle contractions were similar to those obtained for the other hip joint conditions at $30^{\circ}$ of knee flexion. In particular it can be observed that, for any hip joint angle when the initial knee joint angle was $30^{\circ}$, the effect of RF contraction was not to flex the hip but to extend it instead.

\section{Discussion}

Several authors $[12,13,14,15]$ have analysed the effects of isolated muscle contractions by computing the induced acceleration and by using musculoskeletal models similar to the one developed originally by Delp and colleagues $[16,17]$. 
Our work differs methodologically from previous reports in several aspects: we did not just consider the induced acceleration, which represents the tendency to move when instantaneous muscle forces are applied to the system, but we have implemented a complete forward dynamics approach. In this way it was possible to track the movement resulting from an injection of mechanical work for the whole time of the muscle contraction and analyse the final system configuration. Furthermore not only single muscle contractions but combinations of them were simulated. To take into account the complex kinematics of the knee joint a crossing bars linkage was adopted to reproduce the tibio-femoral physiological movement $[5,6,8]$. This was important, if we consider that the forward gliding of the tibia obtained by the crossing bars mechanism when the knee was flexed at $90^{\circ}$ was approximately $3 \mathrm{~cm}$, and this was directly translated into an increase of the SM muscle length by approximately the same amount. This increase had not been obtained by a fixed single axis joint. The use of MR imaging to define insertion points of muscles and ligaments and to design the wrap surfaces insures about the correctness of the modelled lines of action and lever arms of muscles. This is fundamental in particular when the resulting movement depends on the balance between agonists and antagonists muscles. Finally, we did not keep the joint configurations at certain gait phases as a reference, but we systematically analysed various joint configurations of the lower limb, to obtain a more general insight into muscle function and interplay. The muscle contractions as well were considered hypothetical, and they were set to produce the same initial force and muscle shortening, irrespectively of the individual muscle size, in order to compare their dynamical effect. The values adopted ( $25 \mathrm{~N}$ and $0.02 \mathrm{~m}$ respectively) are within the range of feasibility for the muscles considered $[10,11]$. The effect of SM contraction on hip and knee joint motion depended on whether the proximal or the distal segment offered the greater resistance to movement. The flexor 
effect on the knee joint was greater when the foot was free and the pelvis fixed, while SM contraction produced a predominantly extensor effect at the hip joint when the foot was fixed and all the above segments were free to move. The addition of VA contraction considerably enhanced the extensor effect at the hip joint in all conditions. Thus co-contraction of knee extensors and hamstrings may be a mechanism to compensate for weakness of the monoarticular hip extensors and may be a well coordinated, not necessarily pathological action.

Some of our results correspond to those noted by others, particularly Neptune and colleagues [14]. We confirmed that RF can have, for particular knee joint angles an extensor effect at the hip, despite its action as a hip flexor (Figure 4). It is quite likely that the forward acceleration of the shank produces a backward reaction at the knee joint, which in turn produces an extension moment at the hip which prevails over the flexion moment produced by the RF. This is an example of what is called 'dynamic coupling' [18]. Another example, already described by Kimmel and Schwartz [15], was the hip extension effect of VA contraction. To our knowledge the effect of simultaneous activation of SM, VA and RF in a model of this type has not been described previously. We found that, by the addition of knee extensors contraction to SM contraction, the knee movement changed from flexion to extension, and hip extension also increased considerably in all the conditions tested. This confirms our hypothesis that co-contraction of hamstrings and quadriceps can be used to increase hip extensors function. It is not clear if the hip extension produced by SM contraction could be useful to enhance the extensor function of RF at the knee. This seems to be the case in the $30^{\circ}$ hip flexion $/ 30^{\circ}$ knee flexion condition (Figure 4). Knee extension was slightly greater when SM and RF were contracted simultaneously than when RF was contracted alone. However, in all other conditions RF and SM contractions seem to cancel each other out, and the resulting movement was small even after prolonged 
simulation. Interestingly the knee extension movement produced by VA contraction in some initial conditions, particularly at $90^{\circ}$ of hip flexion, was enhanced when SM was contracted simultaneously. As the VA does not cross the hip joint this effect can be attributed to the dynamic coupling of thigh and shank: increased hip extension, produced by SM, would yield a backward acceleration of the thigh that in turn would induce an increased knee extension.

In conclusion it appears that co-contraction of knee extensors and hamstrings enhances the hip extensor effect of the hamstrings, both in conditions where high external forces are applied (see standing upright simulations with pelvis or foot fixed in space), and when the dynamic coupling is involved. In contrast the model showed that, in some situations, hamstring contraction had the potential to enhance the knee extensor effect of RF, although in many simulations this effect was mild or not likely to be relevant clinically.

From a functional point of view we can conclude that co-contraction of hamstrings and knee extensors in conditions of hip extensors weakness or knee flexion may be a strategy to obtain the required extension moment at the hip joint.

\section{References}

1) Sutherland $\mathrm{DH}$, Santi M, Abel MF. Treatment of stiff-knee gait in cerebral palsy: a comparison by gait analysis of the distal rectus femoris transfer versus proximal rectus release. J Pediatr Orthop 1990; 10:433-441.

2) Stewart C, Postans N, Schwartz MH, Rozumalski A, Roberts AP. An investigation of the action of the hamstring muscles during standing in crouch using functional electrical stimulation (FES). Gait Posture 2008; 28:372-377. 
3) Brunner R, Romkes J. Abnormal EMG muscle activity during gait in patients without neurological disorders. Gait \& Posture 2008; 27:399-407.

4) Brunner R, Dreher T, Romkes J, Frigo C. Effects of Plantarflexion on Pelvis and Lower Limb Kinematics. Gait \& Posture 2008; 28:150-156.

5) Pavan EE, Taboga P, Frigo C. Feasibility of a new - joint constrained - lower limb model for gait analysis application. Gait \& Posture 2006; 24(suppl2):20-21.

6) Pavan EE, Taboga P, Frigo C. A mobile axis knee joint model for gait analysis applications. J Biomech 2006; 39(suppl1):501-502.

7) Mommersteeg TJA, Blankevoort L, Huiskes R, Koolos JGM, Kauert JMG. Characterization of the mechanical behaviour of human knee ligaments: a numerical-experimental approach. Journal of Biomechanics 1996; 29(2):151-160.

8) Frigo C, Pavan EE, De Momi E. Musculoskeletal modelling and movement analysis in preoperative surgical planning. Proc Fourth Annual International Conference on Computer Assisted Orthopaedic Surgery Chicago (IL), June 2004:287-288.

9) Clauser CE, McConville JT, Young JW. Weight, volume, and centre of mass of segments of the human body, AMRL Technical Report (TR-69-70). WrightPatterson Air Force Base, OH, 1969.

10) Ralston HJ. Dynamic features of human isolated voluntary muscle in isometric and free contractions. J Appl Physiol 1949; 1(7) :526-533.

11) Yamaguchi GT, Sawa AGU, Moran DW, Fessler MJ, Winters JM. A survey of musculotendon actuator parameters, In: Winters JM, Woo SLY (ed), Multiple Muscle Systems- Biomechanics and Movement Organization, Springer-Verlag, New York, 1990 :716-773. 
12) Kepple T, Siegel KL, Stanhope SJ. Relative contribution of the lower extremity joint moments to forwad progression and support during gait. Gait \& Posture $1997 ; 6: 1-8$

13) Anderson FC, Pandy MG. Individual Muscle Contributions to Support in Normal Walking. Gait \& Posture 2003; 17:159-169.

14) Neptune RR, Zajac FE, Kautz SA. Muscle force redistributes segmental power for body progression during walking. Gait \& Posture 2004; 19:194-205.

15) Kimmel SA, Schwartz MH. A baseline of dynamic muscle function during gait. Gait \& Posture 2006; 23 (2):211-221.

16) Delp SL, Loan JP, Hoy MG, Zajac FE, Topp EL, Rosen JM. An interactive graphics-based model of the lower extremity to study orthopaedic surgical procedures. IEEE Trans BME 1990; 37:757-767.

17) Jonkers I, Stewart S, Spaepen A. The study of muscle action during single support and swing phase of gait: clinical relevance of forward simulation techniques. Gait \& Posture 2003; 17(2):97-105.

18) Zajac FE, Neptune RR, Kautz SA. Biomechanics and muscle coordination of human walking.Part I: Introduction to concepts, power transfer, dynamics and Simulations, Gait and Posture 2002; 16 :215-232.

\section{Legend of figures}

\section{Figure 1}

The dynamic model. The bone models of pelvis, femur, tibia, fibula and patella are included in the geometric solids representing pelvis, thigh and shank. On the right side the anterior and posterior cruciate linkage is represented by hatched lines. 
Figure 2

Above: configuration assumed by the model at initial position ad after simulation of muscle contractions: $\mathrm{SM}=$ contraction of Semimembranosus; $\mathrm{SM}+\mathrm{VA}=$ Semimembranosus and Vasti simultaneously; (pf) = pelvis fixed in space; $(\mathrm{ff})=$ foot fixed in space; below: difference between the joint angles at the end of simulation and the joint angles at the beginning. Positive angles mean flexion increase (flexion movement), negative values mean flexion decrease (extension movement).

\section{Figure 3}

Different configurations assumed by the model after simulation of various single muscle contractions and combinations, starting from $60^{\circ}$ of hip flexion and $60^{\circ}$ of knee flexion H60K60). The sign + means simultaneous contraction. The time of simulation is reported at the bottom of each diagram.

Figure 4

Changes of joint angles in relation to the initial position caused by different combinations of muscle contractions; positive values mean flexion movement, negative values mean extension movement. The initial configurations of hip and knee joint are reported in the labels above and are coded as follow: H9OK30 = hip flexed at $90^{\circ}$ and knee flexed at $30^{\circ} ; \mathrm{H} 60 \mathrm{~K} 60=$ hip and knee flexed at $60^{\circ} ; \mathrm{H}-15 \mathrm{~K} 30=$ hip extended by $15^{\circ}$ and knee flexed at $30^{\circ}$. 


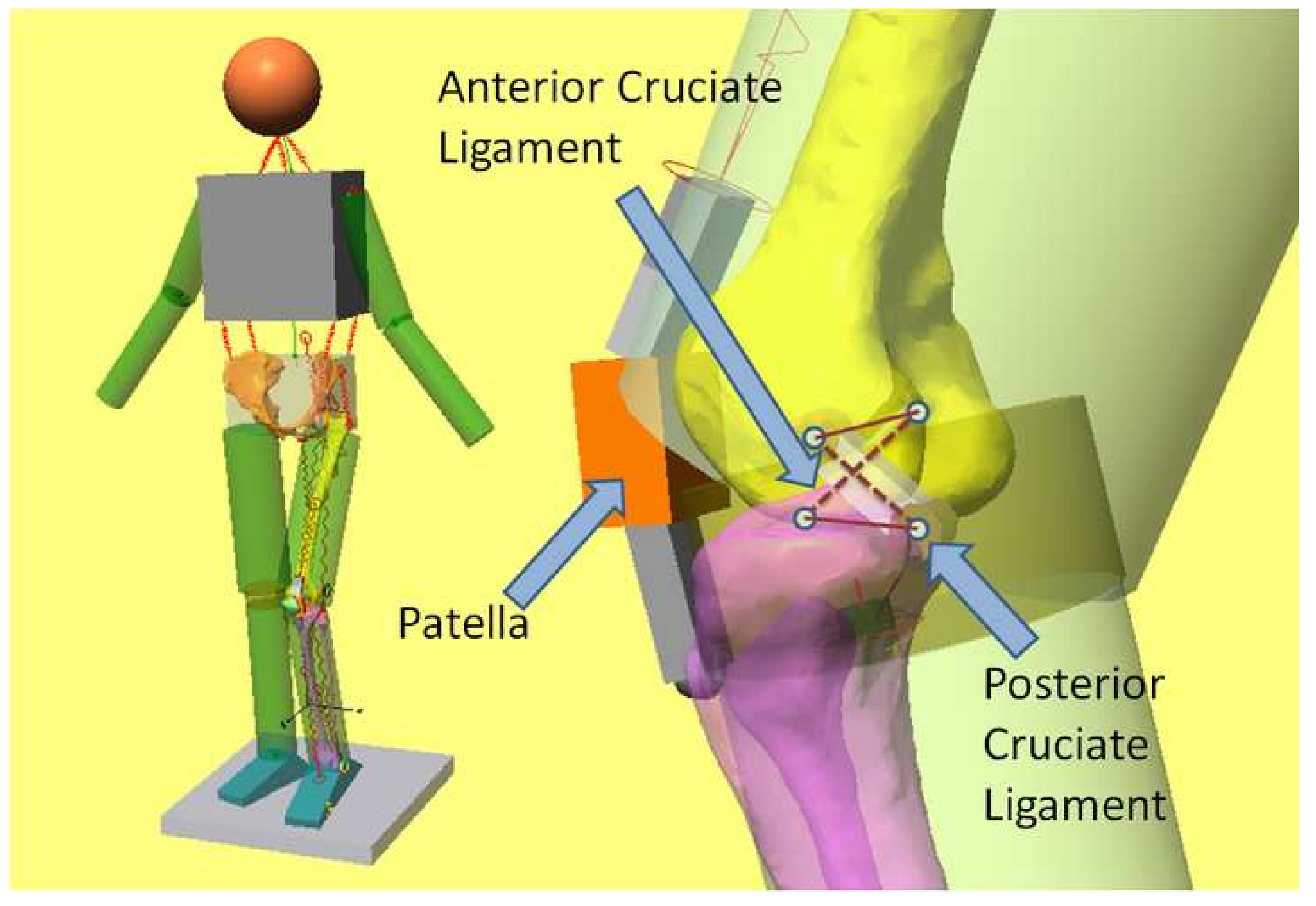

Anterior Cruciate

Ligament

Cruciate Ligament 

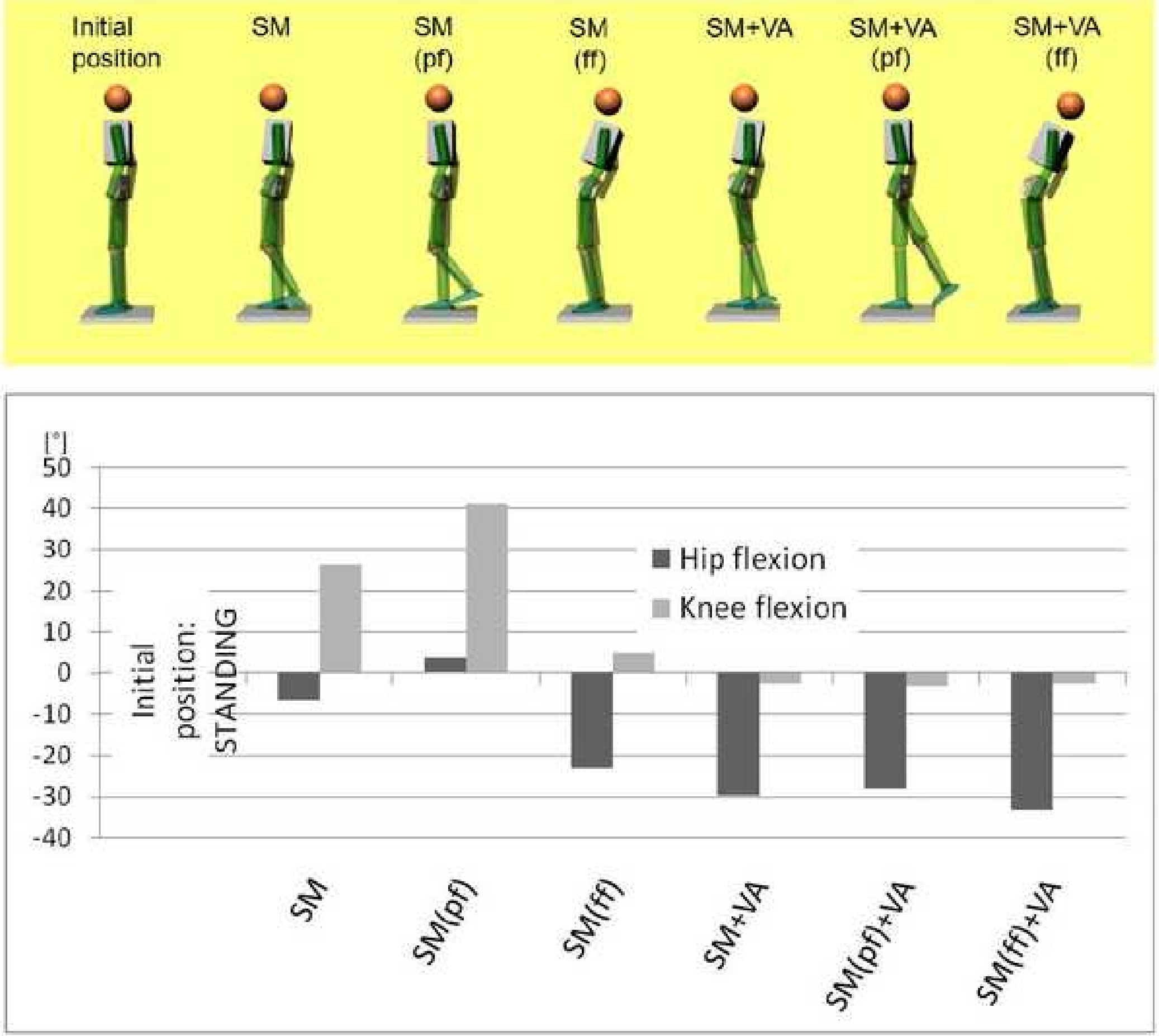


$$
\begin{gathered}
1-1 \\
11 \\
11
\end{gathered}
$$



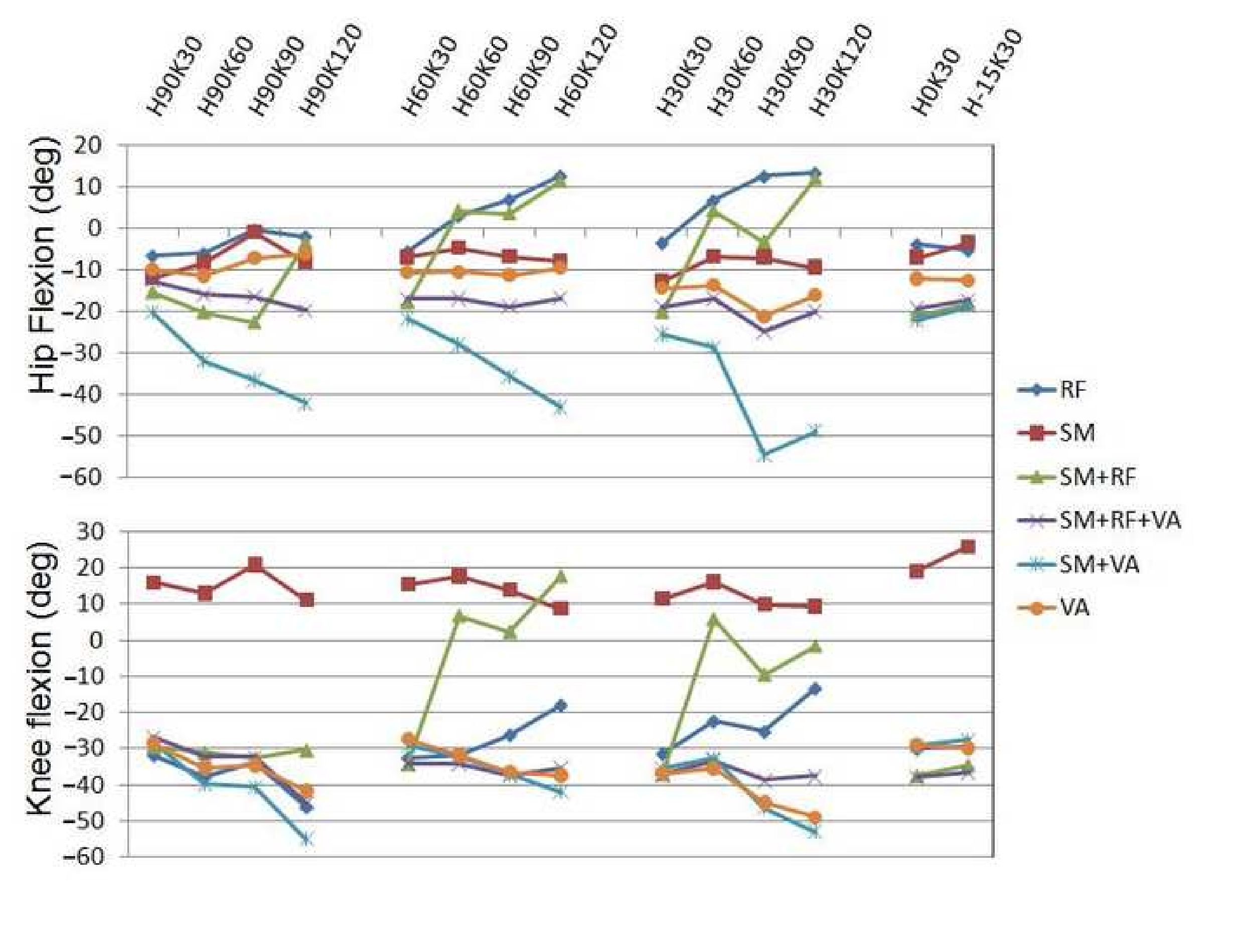